\title{
On the efficiency of bile salt for stable suspension and isolation of single-walled carbon nanotubes-spectroscopic and microscopic investigations
}

\author{
Pawel Lukaszczuk • E. Borowiak-Palen • \\ Mark H. Rümmeli • Ryszard J. Kalenczuk
}

Received: 10 February 2010 / Accepted: 18 May 2010 / Published online: 10 July 2010

(c) The Author(s) 2010. This article is published with open access at Springerlink.com

\begin{abstract}
In this contribution we present a systematic study on the dispersion of SWCNTs in a water-based solution of biocompatible detergent: sodium deoxycholate (DOC). By avoiding harsh chemical conditions, which are known to damage nanotubes structure, a stable SWCNTs suspension was created. Long term stirring of the solution led to preparation of a stable transparent solution, containing welldispersed isolated SWCNTs. The as-prepared dispersion remained stable and clear for two months. Optical absorption spectroscopy was employed to measure SWCNTs suspension stability. Nanotube aggregation was evaluated through the tangential mode ( $\mathrm{G}$ mode) present in the Raman spectrum. High-resolution transmission electron microscopy was employed to observe the mechanism of debundling process.
\end{abstract}

\section{Introduction}

Since carbon nanotubes first being observed [1], due to their great electrical and mechanical properties, they have opened up new opportunities in a wide number of areas of nanotechnology such as electronics, composites and other applications [2, 3]. The origin of these unique features lies in their one-dimensional nature. However, their special properties can be suppressed or entirely quashed when aggregating into bundles. Nanotubes tend to aggregate into large ropes or

P. Lukaszczuk ( $\varangle) \cdot$ E. Borowiak-Palen · R.J. Kalenczuk Institute of Chemical and Environment Engineering, West Pomeranian University of Technology, ul. Pulaskiego 10, 70-322 Szczecin, Poland

e-mail: plukaszczuk@zut.edu.pl

M.H. Rümmeli

Leibniz Institute for Solid State and Materials Research Dresden, Helmholtzstr. 20, 01069 Dresden, Germany bundles via their high surface energy and very strong $\pi-\pi$ interactions. In order to use nanotubes in real applications in technology and medicine, it is necessary to find a simple and inexpensive method for bundle exfoliation into single isolated tubes, thus forming stable dispersions. To get a stable SWCNTs suspension in water one should use certain guest molecules which can reduce the hydrophobic interactions between nanotubes and water. In the literature the most wellknown surfactant is sodium dodecyl sulfate (SDS) which is an anionic detergent used in many cleaning products [4]. Other molecules, such as polymers or DNA, have also been found to act as dispersing agents for nanotubes, however the price of these components is high [5-7]. Dispersion of hydrophobic molecules occurs naturally in the digestion system through the assistance of naturally produced surfactants contained in bile produced by the liver. The great potential of dispersing nanotubes using one of the bile components, sodium deoxycholate (DOC) was reported by Wenseleers et al. [8], however the systematic study on the efficiency of DOC in the formation of stable nanotubes suspension is not provided there. Here, we present a systematic study which leads to the establishment of appropriate protocol for the creation of a stable suspension of SWCNT in a biocompatible aqueous solution of DOC. This avoids any harsh chemical treatment. The relative evaluation of the extent of the debundling of the tubes is also provided. Sodium deoxycholate is a salt formed by $3 \alpha, 12 \alpha$-dihydroxy- $5 \beta$-cholanate (deoxycholic acid) and sodium ion. Optical absorption spectroscopy (OAS) was applied to examine the nanotubes stability in the suspension. Raman spectroscopy was utilized to evaluate the success of the exfoliation procedure. Highresolution transmission electron microscopy (HR-TEM) observation of the analyzed samples was applied for visualization of the exfoliation process. Schematic representation 


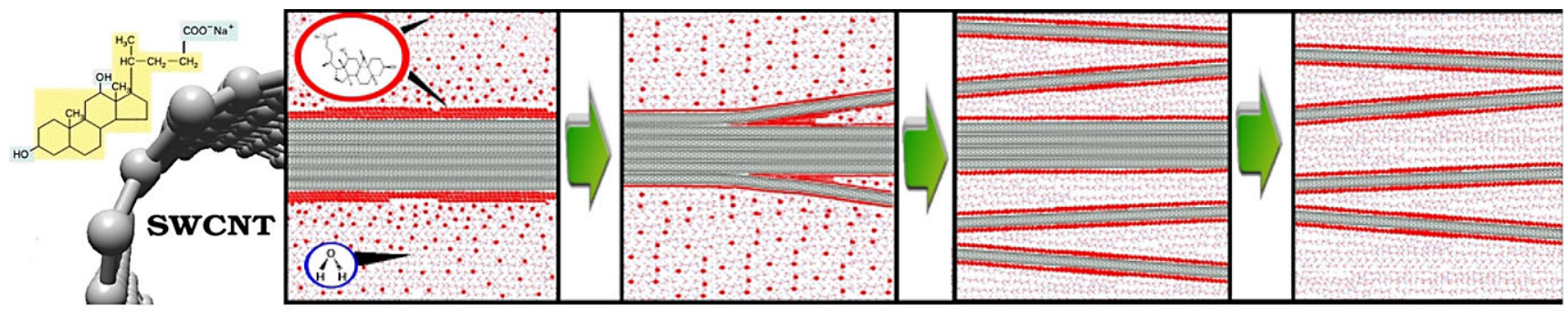

a

b

Fig. 1 a Schematic representation of surfactant attached to SWNCT. Hydrophobic part of the surfactant is indicated by yellow color, hydrophilic groups are indicated by blue color. b Schematic representation of the debundling process

of the surfactant structure and its possible interaction with nanotube is shown in Fig. 1.

\section{Experimental}

Raw SWCNTs used in this experiment were synthesized via the laser ablation technique using $\mathrm{Pt} / \mathrm{Re} / \mathrm{Rh}$ as catalysts [9]. The purification of the raw SWCNTs was accomplished via the following route: (1) annealing in air for $1 \mathrm{~h}$ at $300^{\circ} \mathrm{C}$, (2) refluxing in diluted aqua regia for $24 \mathrm{~h}$, (3) annealing at $1000^{\circ} \mathrm{C}$ under high vacuum $\left(\sim 10^{-5} \mathrm{mbar}\right)$. The second annealing step was performed in order to remove the functional groups and defects introduced to the nanotubes during the acid treatment [10]. Afterwards, the material was filtrated and washed with distilled water. This material we term the reference sample. $3 \alpha, 12 \alpha$-Dihydroxy- $5 \beta$-cholanic acid sodium salt (DOC) was purchased from Sigma-Aldrich with $\geq 97 \%$ purity and was used as supplied. An aqueous SWCNTs solution with $1 \%$ DOC was then prepared. Afterwards, the suspension was placed onto a magnetic stirrer for 17 days. During the mixing procedure, samples were collected for optical absorption and Raman analysis with time intervals of 5, 10, 12, 15 and 17 days. After the dispersion process, the suspensions of the nanotubes were stored at room temperature and the regular monitoring of the integrated area of the optical absorption spectroscopic (OAS) signals, as a measure of the suspension stability, was performed, for 35 days. Typically the SWCNTs OAS spectrum shows three absorption peaks (van Hove singularitiesvHS) correlated to the gaps between energy levels of the density of states. Two of these peaks at about 0.6 and $1.2 \mathrm{eV}$ originate from the semiconducting nanotubes and they are assigned to $\mathrm{E}_{11} \mathrm{~S}$ and $\mathrm{E}_{22} \mathrm{~S}$. The third peak, at about $1.8 \mathrm{eV}$ comes from the metallic nanotubes $\left(\mathrm{E}_{11} \mathrm{M}\right)$. The exact energies of vHS depend on the nanotubes diameter distribution. The OAS response of SWCNTs also allows one to determine the relative nanotube abundance in the sample based on the area under the peaks [11]. Additionally, following
B. Zhao et al. [12] the extinction coefficients of our nanotubes solutions have been determined. According to Beer's law the relationship of the absorbancies of $\mathrm{E}_{22}{ }^{\mathrm{S}}$ and $\mathrm{E}_{11} \mathrm{M}$ and the defined molar concentrations of the reference sample the extinction coefficient can be expressed by:

$A=\varepsilon \times C \times l$

where $A$ absorbance, $\varepsilon$ molar extinction coefficient $\left(\mathrm{L} \times \mathrm{mol}^{-1} \times \mathrm{cm}^{-1}\right), C$ defined concentration of reference sample solution $(\mathrm{mol} / \mathrm{L}), l$ path length of the spectroscopic cuvette $(1 \mathrm{~cm})$.

Optical absorbance spectroscopy was performed using a Jasco V-570 UV/Vis/NIR spectrometer. Samples after each step of preparation were investigated via Raman spectroscopy. In the Raman spectrum the most important mode for the evaluation of the bundling/debundling of SWCNTs effect is the $G$-mode placed between 1500 and $1600 \mathrm{~cm}^{-1}$. In the case of SWCNTs this mode composes of two components: lower energy $G^{-}$(asymmetric peak with a BreitWigner-Fano (BWF) line shape) associated with vibrations along circumferential direction and higher energy $G^{+}$(symmetric peak with a Lorentzian line shape) correlated with stretching vibrations along the tube axis. In this case, the disappearance/appearance of the BWF is attributed to the debundling/bundling of the tubes. Raman spectra were obtained with a Renishaw InVia Raman Microscope spectrometer with $785 \mathrm{~nm}$ excitation laser wavelength. The morphology of the samples was investigated via high-resolution transmission electron microscopy (HR-TEM) (FEI Tecnai F20 $(200 \mathrm{kV}))$. The specimen for HR-TEM was prepared with a single drop of the analyzed suspension placed on the holey carbon filmed grids at room temperature. Optical and vibrational response of SWCNTs can be strongly dependent on the nanotube-surfactant interaction leading to a charge transfer or an electronic polarization [13-15]. Therefore, in order to confirm the stability of the created suspension turbidity measurements were also conducted (data not shown here) [16].

Figure 2a presents OAS spectra of the investigated dispersions (molar concentration given in the inset) and Fig. $2 b$ 

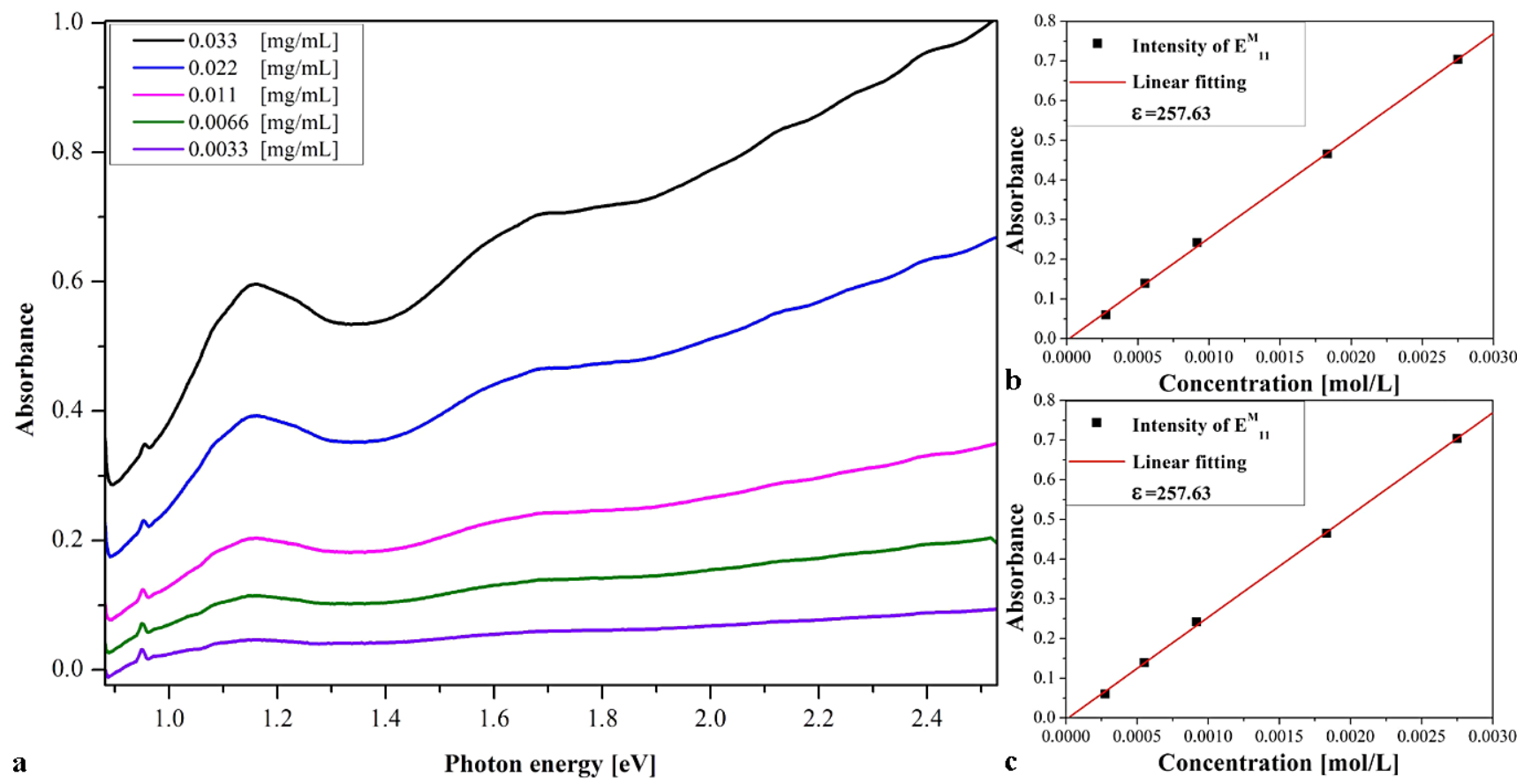

Fig. 2 a OAS spectra of nanotubes reference solutions. b, c Relationship between absorbance and molar concentration of nanotubes based on $\mathrm{E}_{22} \mathrm{~S}$ and $\mathrm{E}_{11}{ }^{\mathrm{M}}$ peaks

and $2 \mathrm{c}$ provide the estimated linear relationship between molar concentration of the nanotubes solution versus its absorbance determined basing on $\mathrm{E}_{11}{ }^{\mathrm{M}}$ and $\mathrm{E}_{22} \mathrm{~S}$, respectively. The exact values of the extinction coefficients are given in the insets of these graphs. This relationship obeys the Lambert-Beer law with correlation coefficient equal 0.99971 when absorbance intensity of $\mathrm{E}_{11}{ }^{\mathrm{M}}$ was used and 0.99965 when absorbance intensity of $\mathrm{E}_{22} \mathrm{~S}$ was used.

These curves have been applied to estimate the concentration of the optimally dispersed nanotubes suspension.

\section{Results and discussion}

After each step of preparation, before and after purification processes (acid treatment and annealing) the material was investigated by Raman spectroscopy in order to estimate the $G / D$ ratio (Fig. 3). The $G / D$ ratio is an indicator of the sample quality in respect of number of defects in the graphite structure. Lower $G / D$ ratio in the refluxed sample in comparison to the annealed one indicates that acid treatment introduces defects into nanotubes wall structure. After the annealing process, $G / D$ ratio increased up to 8.85 what is even higher than for the raw material $(G / D=7.13)$. A higher $G / D$ ratio proves that the tubes quality is better.

Initial OAS measurements were performed to evaluate the time required to form a stable DOC-SWCNTs suspension (Fig. 4). Here, the integrated area of the optical bands

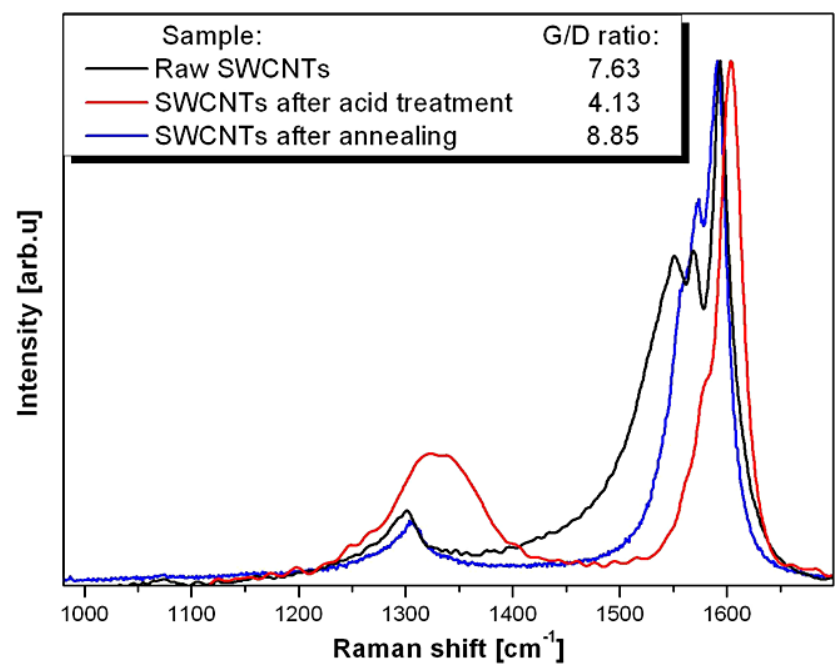

Fig. 3 Raman spectra in range of the $D$ and $G$ mode of the tree samples: raw, after acid treatment and after annealing

of SWCNTs indicates that after 10 days of the stirring, the concentration of the tubes does not change significantly. The optimal stirring time (after which no change in the integrated area was registered) is 17 days. Thus, the stability of the solution stirred for 17 days was estimated. Additionally, it is worth noting that the presence of the van Hove singularities in the optical spectrum of the solution indicates that nanotube/DOC interaction (via an electronic polarization) is not sufficiently strong to smear out the vHS, which usually 
Fig. 4 Left panel: OAS spectra of the stirred solution (HR-TEM image of the reference sample as an inset), right panel: ratio and the area of $\mathrm{E}_{22} \mathrm{~S}$ and $\mathrm{E}_{11} \mathrm{M}$ peaks in the samples
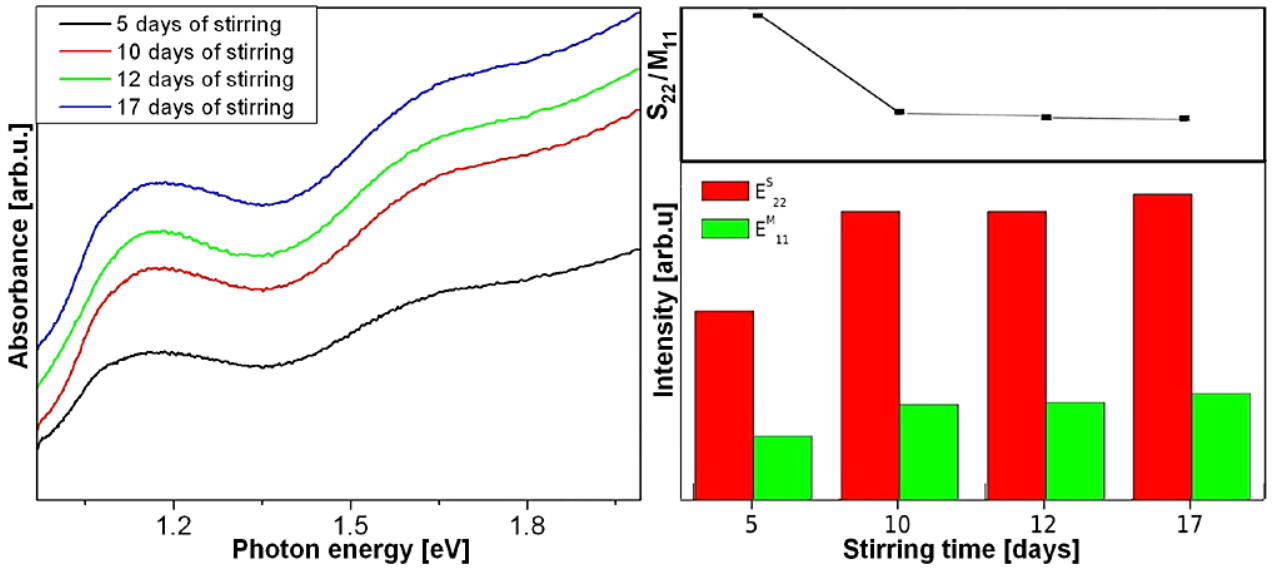

Fig. 5 Left panel: OAS spectra of the supernatant, right panel: ratio and the area of $\mathrm{E}_{22}{ }^{\mathrm{M}}$ and $\mathrm{E}_{11}{ }^{\mathrm{M}}$ peaks in the samples
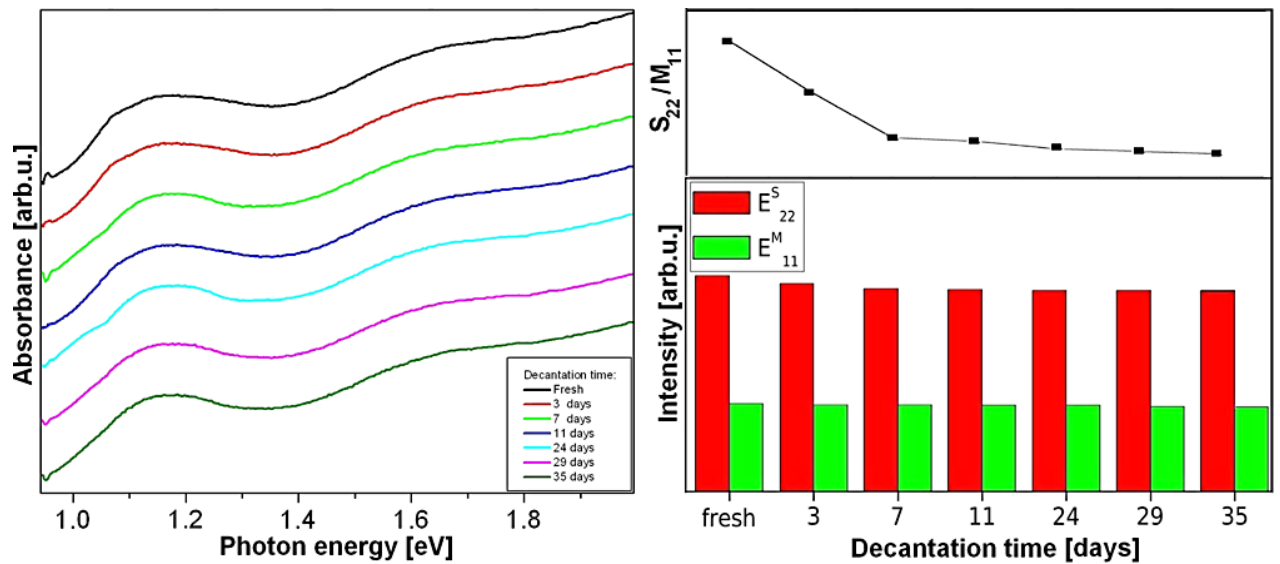

occur if charge transfer takes place [13-15]. The full disappearance of the optical bands due to a Fermi level shift was previously reported [17].

In the next step the DOC/SWCNTs solution (17 days stirring) was transferred to a quartz cuvette and placed in the UV/Vis/NIR spectrometer to decant. OAS spectra of the supernatant collected over various time intervals are indicated in Fig. 5 (right panel- $x$ axis).

The sample was introduced directly after the stirring to the measurement chamber in order not to induce any additional vibration which could enhance the SWCNTs abundance. Analyzing the OAS spectra one can clearly see that the integrated area of optical response of the supernatant during the decantation for 3 days dropped about $8 \%$. After 7 days the integrated area dropped by $2 \%$. However, after that time it remained stable, even after two months. A very weak oscillation of the peaks area is visible on the OAS spectra after a decantation time above 7 days $( \pm 1 \%$ of the total area). This can be caused by the environment (e.g. temperature and pressure changes). The OAS measurements spanned over 35 days. The data are presented in Fig. 5 (left panel). The OAS data suggest that the optimal protocol to form a stable suspension of SWCNTs in DOC solution is the decantation for 7 days followed by stirring for 17 days.
In case of our study the concentration of nanotubes in this suspension was calculated using the obtained molar extinction coefficients and was equal to $0.429 \mathrm{mM}$ (basing on $\mathrm{E}_{22} \mathrm{~S}$ and $\mathrm{E}_{11}{ }^{\mathrm{M}}$ ).

As mentioned in the experimental section the appearance/disappearance of $G^{-}$with its Breit-Wigner-Fano line shape of SWCNTs observed in Raman spectroscopy arises from bundling/debundling effects $[18,19]$ or it can be used to estimate the metallic/semiconducting tubes content, this latter point is not an issue in our study. Figure 6 (left panel) presents the $G$ mode region of Raman spectra of the reference sample (in a solid), optimally dispersed sample (in a solution) and optimally dispersed sample in the form of the solid film.

A dramatic decrease of the $G^{-}$band intensity is observed in the case of the suspended SWCNTs with respect to the reference sample or the suspended sample after the drying to a solid film. This indicates an efficient debundling in the suspension. In order to relatively quantify the number of debundled tubes in the samples, the ratio of areas of the high-frequency component of the $G$ band to the lowfrequency component $\left(A_{G+} / A_{G-}\right)$ for these three samples was calculated and the results are presented in Fig. 6 (middle panel). This ratio serves as a measure of nanotubes ag- 
Fig. 6 Left panel: $G$ mode region of Raman spectra of the reference sample in the solid film (black line), the suspended solution of DOC/SWCNTs (red line) and this solution after drying (blue line). Spectra are normalized to $G^{+}$. Middle panel: distribution of $A_{G+} / A_{G-}$ in the analyzed samples. Right panel: the images of the solution before and after application of the suspending protocol
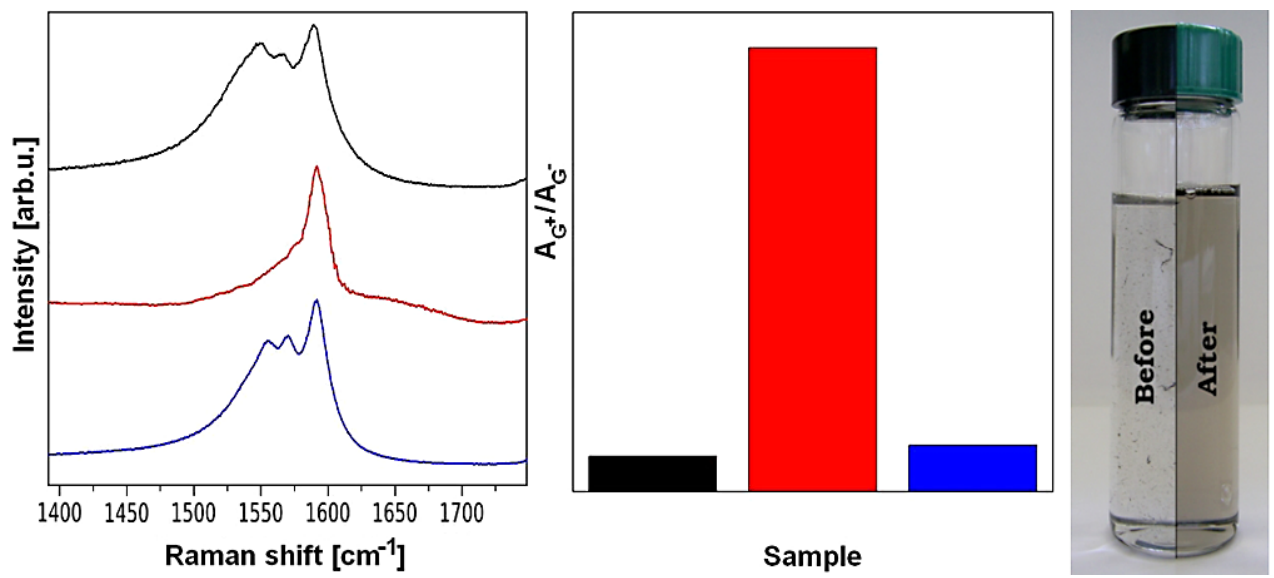

Fig. 7 TEM images taken from: a reference sample, b solution during the stirring, c solution after the stirring, d supernatant after 35 day of decantation
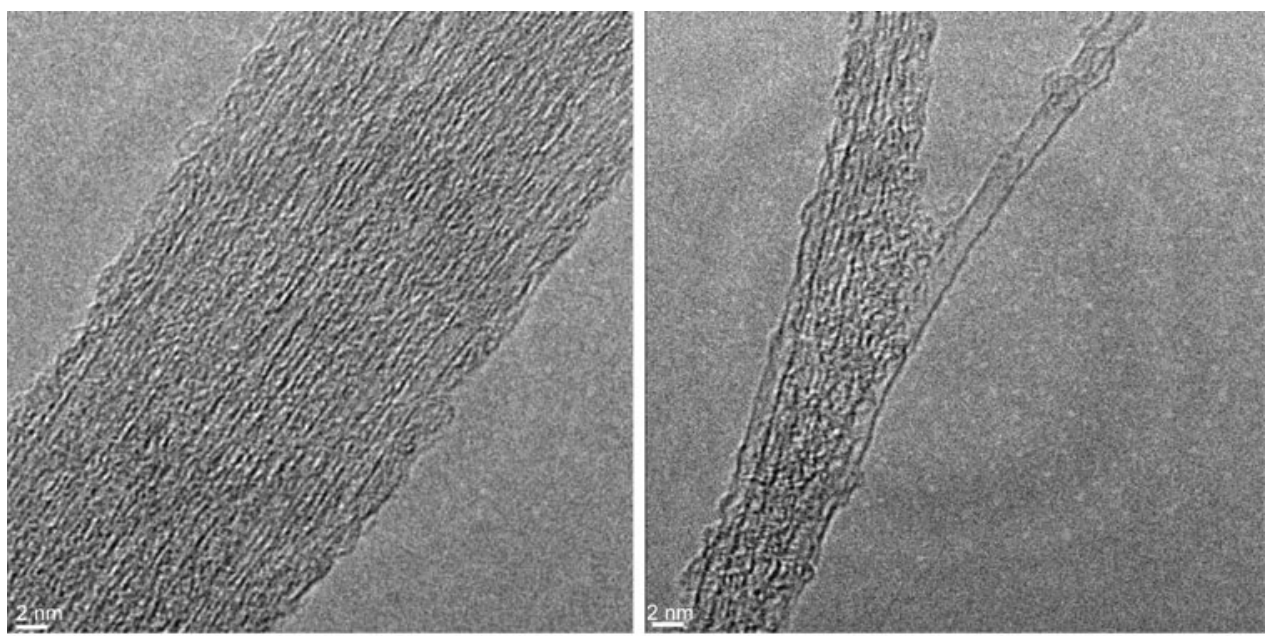

a
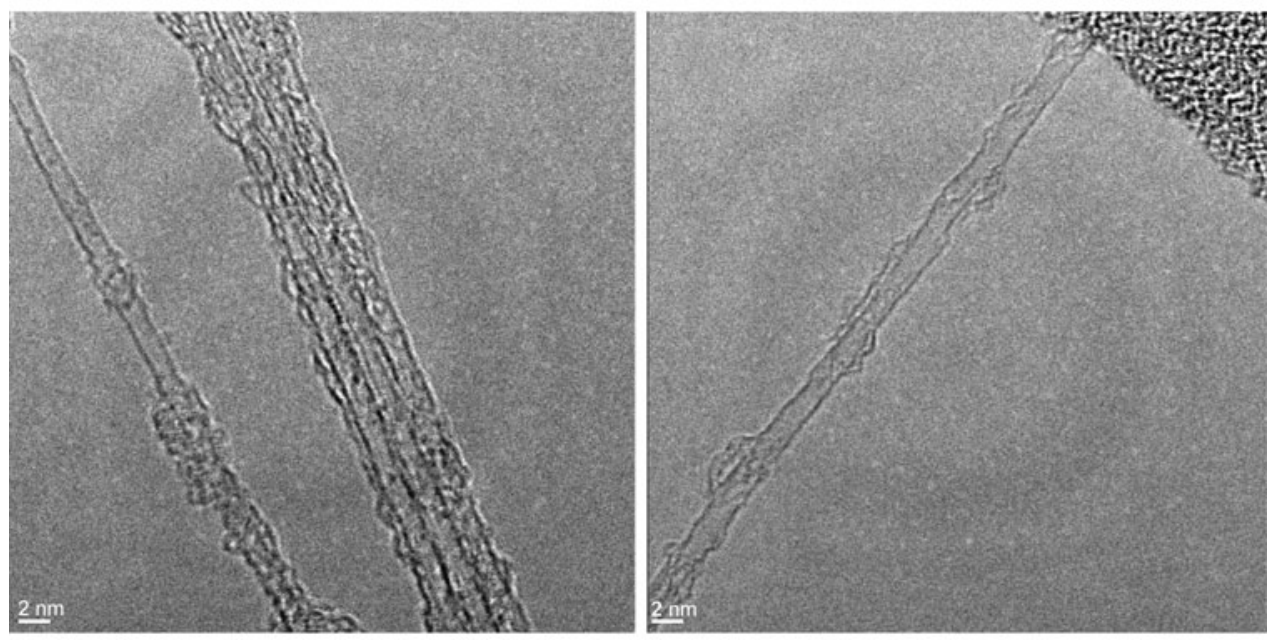

d gregation into bundles and it ranges from relatively higher values for SWCNTs suspended in our solution to lower values observed in the Raman spectrum of the reference SWCNTs in the solid state. [20, 21]. One can also notice that the area under the $G^{-}$of the samples measured after drying the suspended sample is not recovered to its initial value. This would indicate that the bundle size of this sample is significantly smaller or a fraction of the tubes remain debundled. To confirm this statement microscopic studies are required. The BWF line shape in $G$ mode of SWCNTs can also be 
influenced by the surfactant/nanotubes interaction. Due to the fact that DOC has two hydroxyl groups in its molecular structure one could expect charge transfer to the nanotubes (however, the effect of electron doping is not significant as the OAS spectra show no reduction in the $\mathrm{E}_{11}{ }^{\mathrm{M}}$ peak relative to the $\mathrm{E}_{22} \mathrm{~S}$ peak, which occurs when the Fermi level shifts in doped systems). Hence, one can conclude that the bundling effect dominates, and therefore the BWF lineshape can be successfully applied for the evaluation of the degree of debundling in our system.

Additionally, the digital camera images of the solution before and after application of the optimized protocol for the formation of the stable DOC/SWCNTs suspension are presented in the right panel of Fig. 6. One can clearly see that the suspension is fully homogeneous and the OAS confirmed that it remains remarkably stable, even after two months.

HR-TEM study was performed in order to visualize the debundling process (Fig. 7). However, for this purpose this technique has certain limitation due to the rebundling of the individual tubes during the preparation of TEM specimen. Figure 7 a presents a typical bundle of the carbon nanotubes (diameter $\sim 24 \mathrm{~nm}$ ) after the purification process (reference sample). Our systematic observations of the sample revealed the bundle size was in the range between 14$40 \mathrm{~nm}$. Figure $7 \mathrm{~b}$ shows the typical morphology of the bundles during the stirring process. Here, one can clearly see the way the surfactant debundles and partially isolates the single walled carbon nanotubes from its bundled state. In the sample fraction obtained after the full stirring procedure is shown in Fig. 7c. This material contains very thin bundles (up to 4 tubes) and individual tubular structures. As a consequence of the sedimentation process the remaining supernatant was strongly enriched with an abundance of isolated carbon nanotubes (Fig. 7d). Due to the preparation of TEM grid at room temperature the amorphous structure detected around the tubes can be attributed to the decomposed DOC. Those observations are in full agreement with spectroscopic evaluation of the stable suspension and isolation of single walled carbon nanotubes.

\section{Conclusion}

We have presented a straightforward protocol to create a stable suspension of SWCNTs in a biocompatible surfactant: DOC aqueous solution. The procedure is not aggressive. Analysis of the OAS and Raman spectra show that sodium deoxycholate has a great potential for SWCNTs dispersion as well for the debundling into individual tubes. The spectroscopic analysis was confirmed by HR-TEM investigations.
DOC is a natural compound, which is present in a human body and it can be potentially used for medical applications such in the drug delivery systems development.

Acknowledgements The research presented here was sponsored by Polish Ministry grant funded in 2009-2012.

Open Access This article is distributed under the terms of the Creative Commons Attribution Noncommercial License which permits any noncommercial use, distribution, and reproduction in any medium, provided the original author(s) and source are credited.

\section{References}

1. S. Iijima, Nature 354/6348, 56 (1991)

2. H. Dai, Surface Sci. 500, 218 (2002)

3. L. Ci, J. Suhr, V. Pushparaj, X. Zhang, P.M. Ajayan, Nano Lett. 8(9), 2762 (2008)

4. K. Yurekli, C.A. Mitchell, R. Krishnamoorti, J. Am. Chem. Soc. 126(32), 9902 (2004)

5. H. Cathcart, V. Nicolosi, J.M. Hughes, W.J. Blau, J.M. Kelly, S.J. Quinn, J.N. Coleman, J. Am. Chem. Soc. 130(38), 12734 (2008)

6. G. Dukovic, M. Balaz, P. Doak, N.D. Berova, M. Zheng, R.S. Mclean, L.E. Brus, J. Am. Chem. Soc. 128(28), 9004 (2006)

7. J.F. Campbell, I. Tessmer, H. Holden Thorp, D.A. Erie, J. Am. Chem. Soc. 130(32), 10648 (2008)

8. W. Wenseleers, I.I. Vlasov, E. Goovaerts, E.D. Obraztsova, A.S. Lobach, A. Bouwen, Adv. Funct. Mater. 14, 1105 (2004)

9. M. Rummeli, M. Loffler, C. Kramberger, F. Simon, F. Fulop, O. Jost, R. Schonfelder, A. Gruneis, T. Gemming, W. Pompe, B. Buchner, T. Pichler, J. Phys. Chem. C 111, 4094 (2007)

10. E. Kowalska, P. Kowalczyk, J. Radomska, E. Czerwosz, H. Wronka, M. Bystrzejewski, J. Therm. Anal. Calorim. 86(1), 115-119 (2006)

11. E. Borowiak-Palen, T. Pichler, X. Liu, M. Knupfer, A. Graff, O. Jost, W. Pompe, R.J. Kalenczuk, J. Fink, Chem. Phys. Lett. 363, 567 (2002)

12. B. Zhao, M.E. Itkis, S. Niyogi, H. Hu, J. Zhang, R.C. Haddon, J. Phys. Chem. B 108, 8136 (2004)

13. M. Shoda, S. Bandow, Y. Maruyama, S. Iijima, J. Phys. Chem. C 113(15), 6033 (2009)

14. J. Wang, Y. Li, J. Am. Chem. Soc. 131(15), 5364 (2009)

15. P.J. Boul, D.G. Cho, G.M.A. Rahman, M. Marquez, Z. Ou, K.M. Kadish, D.M. Guldi, J.L. Sessler, J. Am. Chem. Soc. 129(17), 5683 (2007)

16. D. Nepal, S. Balasubramanian, A.L. Simonian, V.A. Davis, Nano Lett. 8(7), 1896 (2008)

17. K. Seo, K.A. Park, C. Kim, S. Han, B. Kim, Y.H. Lee, J. Am. Chem. Soc. 127(45), 15724 (2005)

18. J.L. Blackburn, C. Engtrakul, T.J. McDonald, A.C. Dillon, M.J. Heben, J. Phys. Chem. B 110, 25551 (2006)

19. V.A. Karachevtsev, A.Yu. Glamazda, U. Dettlaff-Weglikowska, V.S. Leontiev, P.V. Mateichenko, S. Roth, A.M. Rao, Carbon 44, $1292(2006)$

20. C. Jiang, K. Kempa, J. Zhao, U. Schlecht, U. Kolb, T. Basché, M. Burghard, A. Mews, Phys. Rev. B 66, 161404 (2002)

21. N. Bendiab, R. Almairac, M. Paillet, J.L. Sauvajol, Chem. Phys. Lett. 372(1), 210 (2003) 